\title{
Effects of Continuous Positive Airway Pressure Treatment on Cognitive Functions in the Korean Elderly with Obstructive Sleep Apnea
}

\author{
Suk-Hoon Kang, $\mathrm{MD}^{1,2}$, In-Young Yoon, MD, PhD ${ }^{3,4}$, Sang Don Lee, $\mathrm{MD}^{5}$, Tae Kim, MD, PhD \\ 'Department of Psychiatry, Veterans Health Service Medical Center, Seoul, Korea \\ ${ }^{2}$ Center for Sleep Medicine, Veterans Health Service Medical Center, Seoul, Korea \\ ${ }^{3}$ Department of Neuropsychiatry, Seoul National University Bundang Hospital, Seongnam, Korea \\ ${ }^{4}$ Department of Psychiatry, Seoul National University College of Medicine, Seoul, Korea \\ ${ }^{5}$ Department of Psychiatry, Korean Armed Forces Capital Hospital, Seongnam, Korea \\ ${ }^{6}$ Department of Psychiatry, Kyung Hee University Hospital at Gangdong, Seoul, Korea
}

Background and Objective Obstructive sleep apnea (OSA) in elderly is accompanied by cognitive decline. However, it is not well known whether these deleterious changes can be reversed by continuous positive airway pressure (CPAP) treatment. Therefore, we aimed to elucidate the effect of CPAP on cognitive function in the Korean elderly with OSA.

Methods Forty-two patients ( $\geq 60$ yrs) with moderate-to-severe OSA and 41 normal controls were enrolled, and divided into treated subjects with CPAP $(n=28)$ and non-treated subjects $(n=14)$. We performed baseline measurements of cognition using the Korean version of the consortium to establish a registry for Alzheimer's disease neuropsychological battery. Additionally, digit span test, Stroop color and word test (SCW) and executive clock drawing task were applied. About 175.6 days later, second measurements of cognitive function were completed.

Results OSA groups had higher body mass index than normal controls $(t=3.132, p=0.002)$. In comparison of cognition, OSA groups showed more decline in digit span test (forward; $\mathrm{t}=-3.647, \mathrm{p}=0.001$, backward; $\mathrm{t}=-7.925, \mathrm{p}<0.001$ ) with Bonferroni correction. In the OSA group, subjects with CPAP treatment were younger $(t=2.787, p=0.008)$ and had a longer education years $(t=2.419, p=0.020)$ than non-treated subjects. In comparison of cognition after CPAP treatment, they showed significant improvement in the executive function ( $\mathrm{SCW} ; \mathrm{F}=5.660, \mathrm{p}=0.023$ ) after adjusting the age and education years, considering interaction effect with time and groups.

Conclusions Short-term CPAP treatment could improve executive function, but might not be effective on other cognitions in the elderly with OSA. Future study with long-term CPAP is required to evaluate beneficial effects on cognitive dysfunctions in elderly with OSA.

Sleep Med Res 2016;7(1):10-15

Key Words Elderly, Obstructive sleep apnea, Cognitive function, Continuous positive airway pressure.

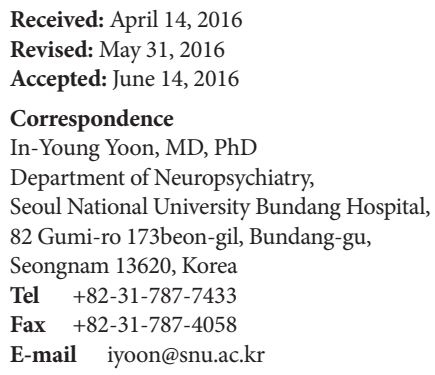

\section{INTRODUCTION}

Obstructive sleep apnea (OSA) is characterized by nocturnal hypoxia and frequent arousal during sleep as a result of repetitive nocturnal respiratory pauses. ${ }^{1}$ In the Wisconsin sleep cohort with stratified random sample of Wisconsin state employees aged 30-60 years, the prevalence of OSA defined as a clinical syndrome was $2 \%$ in women and $4 \%$ in men. ${ }^{2}$ OSA contributes to comorbidities of cardiovascular disease or metabolic dysfunction, and progression of cancer by increasing sympathetic activation with blood pressure elevation and vascular inflammation with endothelial dysfunction. ${ }^{3}$ OSA also has significant neuropsychological problems including stroke, cognitive decline, depression, headaches and peripheral neuropathy. ${ }^{4}$

The effect of OSA on cognition is not well-documented. ${ }^{5}$ Although cognitive decline was unrelated with severity of OSA in some studies, significant impairments in the several neurocognitive assessments such as vigilance, executive functioning, or coordination were reported in the OSA patients, as compared to healthy individuals. ${ }^{6}$ Several different mechanisms of cogni- 
tive dysfunction in patients with OSA are suggested. ${ }^{7,8}$ One mechanism is excessive daytime sleepiness causing cognitive impairments. ${ }^{7}$ Additionally, intermittent hypoxemia is associated with declines of motor and processing speed, spatial abilities and processing speed in individuals with OSA. ${ }^{8}$ But understanding mechanism of cognitive deficit in OSA is complicated due to various clinical and demographic factors to impact cognition. Aging as a risk factor for OSA is associated with other medical conditions. Younger adults with OSA performed better on cognitive tests than older adults with OSA. ${ }^{9}$ Moreover, other sleep disturbances such as profound changes in sleep structure, sleep fragmentation and earlier awakening may be considered as risk factors. ${ }^{10}$

Continuous positive airway pressure (CPAP) normalizes both sleep fragmentation and oxygen desaturation in OSA patients. ${ }^{11}$ Significant improvement of cognitive performance, such as memory, attention and executive function following CPAP treatment after 3 months and 6 months was reported. ${ }^{12,13}$ Case-control designed study also reported improvement of memory and executive function in OSA patients treated with CPAP compared to age-matched healthy controls. ${ }^{14}$ However, previous studies on CPAP treatment for cognition had some limitations. Firstly, various inclusion criteria of apnea-hypopnea index (AHI) cutoff was used to divide study subjects, such as $\mathrm{AHI} \geq 5, \mathrm{AHI} \geq$ 10 , AHI $\geq 20$, or AHI $>30 .{ }^{14-17}$ Some studies selected participants based on the number of respiratory events during sleep irrespective of symptoms and without American academy of sleep medicine (AASM) guideline of study eligibility. ${ }^{15}$ Additionally, larger sample study of 1098 participants diagnosed with OSA did not include older adults. ${ }^{13}$ Meanwhile, in the elderly population studies, small sample size and a different criteria for old adults such as $>55$ years or $>65$ years might be methodological limitations. ${ }^{12,17,18}$ Therefore, the aim of this study was to evaluate the effect of CPAP treatment on cognition in the Korean elderly patients aged $\geq 60$ years with mild to severe OSA.

\section{METHODS}

\section{Study Subjects}

Study participants were 82 treatment-naïve elderly patients aged $\geq 60$ years. Based on clinical interviews and medical records, individuals with neurodegenerative diseases, major depressive disorder or cognitive disorder were excluded. An AHI of $\geq 15$ was considered as diagnostic for OSA. ${ }^{19}$ Two participants had mild to moderate OSA (15-30), and 39 had severe OSA $(\mathrm{AHI} \geq 30)$. The Institutional Review Board of Seoul National University Bundang Hospital approved this study protocol. All study participants provided written informed consent.

\section{Nocturnal Polysomnography and CPAP Titration}

We used an Embla TM N7000 (Embla, Reykjavik, Iceland) and standard electrodes and sensors. Electroencephalography electrodes were applied at C4/A1, C3/A2, O1/A2, and O2/A1, and two electrooculography electrodes were applied at the sides of both eyes to record horizontal and vertical eye movements. Electromyography electrodes were applied at the submentalis muscle and both anterior tibialis muscles recorded limb movements during sleep. Strain gauges were used to record chest and abdominal respiratory movements, and nasal pressure cannulas and thermistor were used to record airflow. Arterial oxygen saturation was measured using a pulse oximeter applied on an index finger. Based on the criteria of Rechtschaffen and Kales, ${ }^{20}$ we scored every 30-second epoch of nocturnal polysomnography. Apnea was defined as the complete cessation of airflow for at least $10 \mathrm{~s}$, and hypopnea was defined as a substantial reduction in airflow (> 50\%) for at least $10 \mathrm{~s}$ or a moderate reduction in airflow for at least $10 \mathrm{~s}$ associated with electroencephalography arousals or oxygen desaturations $(\geq 4 \%){ }^{21} \mathrm{AHI}$ was defined as the total number of apneas and hypopneas per hour of sleep, and the oxygen desaturation index was defined as the number of oxygen desaturations ( $\geq 4 \%$ ) per hour of sleep. The OSA was diagnosed as $\geq 15$ obstructive respiratory events ( $\mathrm{AHI} \geq 15$ ) in this study. After the polysomnographic baseline evaluation, a second night polysomnography was performed in OSA patients for the titration of CPAP. The optimal pressure was the minimum pressure to completely eliminate apneas and hypopneas in each patient. CPAP compliance was calculated days with using CPAP in 4 hours or more of follow up duration.

\section{Clinical Evaluation and Cognition Assessments}

Clinical data were collected using a standardized protocol, including questions about current medical conditions and use of medications. Daytime sleepiness was evaluated using the Epworth sleepiness scale (ESS). Both treated subjects with CPAP treatment and non-treated subjects underwent a complete neuropsychological evaluation with the following test battery. To ensure comprehensive neuropsychological assessments, neuropsychologists administered the Korean version of the consortium to establish a registry for Alzheimer's disease neuropsychological battery (CERAD) and some additional tests. ${ }^{22}$ Memory performance were assessed by the digit span forward (DSF) and digit span backward (DSB), word list recall (WLR) and constructional praxis recall (CR) in CERAD. Executive functions were examined by Stroop color-word test (SCW $)^{23}$ and verbal fluency of CERAD. Visuospatial abilities were performed by the constructional praxis $(\mathrm{CP})$ of CERAD and executive clock drawing task (CLOX2). All participants with OSA performed cognitive function tests at baseline and after 3 months.

\section{Data Analysis}

Intergroup differences between OSA subjects and normal control, or between treated subjects with CPAP and non-treated subjects were assessed using chi-square test and indepen- 
dent $\mathrm{t}$-test. The generalized linear model for repeated measure analysis of variance (ANOVA) test adjusted for age and education years was used to analyze cognitive function measures over time with CPAP treatment. The statistical significance criterion was defined as a p value $<0.05$ for two-tailed test. The level of significance for comparison of multiple neuropsychological tests was set at $\mathrm{p}<0.0063(=0.05 / 8)$ according to Bonferroni correction. SPSS version 21.0 for Windows (SPSS Inc., Chicago, IL, USA) was used for statistical analysis.

\section{RESULTS}

\section{Demographic and Clinical Characteristics}

A total of 82 subjects participated in the study. Participants were divided two groups, OSA groups $(n=41)$ and normal healthy control $(n=41)$ (Table 1$)$. Both groups were age and gender matched. OSA patients composed of 14 non-treated subjects who did not have a CPAP treatment, and 28 treated subjects who underwent CPAP titration examination and took CPAP treatment. Table 1 showed clinical characteristics and neuropsychological parameters of the study subjects. OSA groups had a decline of memory performance (DSF; $\mathrm{t}=-3.647$, $\mathrm{p}=0.001$, DSB; $\mathrm{t}=-7.925, \mathrm{p}<0.001)$ with Bonferroni correction.

\section{Baseline Clinical Characteristics and Polysomnographic Data between Non-Treated Subjects and Treated Subjects}

Non-treated subjects $(\mathrm{n}=13)$ were older $(\mathrm{t}=2.787, \mathrm{p}=0.008)$ and had lower education years $(\mathrm{t}=-2.419, \mathrm{p}=0.020)$ than treated subjects $(\mathrm{n}=28)$ (Table 2$)$. Polysomnographic parameters showed no difference. ESS score showed no difference between groups at baseline $(\mathrm{t}=0.548, \mathrm{p}=0.588)$. After treatment, ESS score within OSA groups showed no significant difference, as compared to pre-treatment $(\mathrm{t}=0.482, \mathrm{p}=0.421)$.

\section{Comparison of Neuropsychological Parameters between Treated and Non-Treated OSA Subjects after CPAP Treatment}

Twenty-eight treated subjects and 13 non-treated subjects were re-examined about 176 days later. After analysis of repeated measures ANOVA with adjustment of age and education, WLR test $(\mathrm{F}=7.599, \mathrm{p}=0.009)$ and SCW $(\mathrm{F}=4.354, \mathrm{p}=$ 0.044 ) revealed significant difference within subjects. No significant interaction effect of time and group was showed except $\operatorname{SCW}(\mathrm{F}=5.660, \mathrm{p}=0.023)($ Table 3$)$.

Table 1. Clinical characteristics and neuropsychological parameters in OSA subjects and normal control subjects

\begin{tabular}{|c|c|c|c|c|}
\hline & OSA $(n=41)$ & Normal $(\mathrm{n}=41)$ & $\mathrm{t} / \mathrm{x}^{2}$ & p-value \\
\hline Age (years) & $66.5(5.2)$ & $66.6(5.1)$ & -0.064 & 0.949 \\
\hline Gender (male) & $28(68.3 \%)$ & $28(68.3 \%)$ & 1.000 & 1.000 \\
\hline Education (years) & $13.7(4.8)$ & $13.4(4.3)$ & 0.242 & 0.810 \\
\hline BMI $\left(\mathrm{kg} / \mathrm{m}^{2}\right)$ & $25.3(3.4)$ & $23.3(2.5)$ & 3.132 & $0.002^{*}$ \\
\hline AHI (events/hr) & $50.1(15.9)$ & $4.8(3.9)$ & 17.780 & $<0.001^{*}$ \\
\hline \multicolumn{5}{|c|}{ Neurocognitive function tests } \\
\hline MMSE-KC & $27.0(3.3)$ & $27.4(1.7)$ & -0.626 & 0.533 \\
\hline \multicolumn{5}{|l|}{ Memory } \\
\hline DSF & $6.5(1.3)$ & $8.1(2.3)$ & -3.647 & $0.001^{\dagger}$ \\
\hline DSB & $4.1(1.6)$ & $6.7(1.3)$ & -7.925 & $<0.001^{\dagger}$ \\
\hline WLR & $5.9(2.2)$ & $5.7(1.7)$ & 0.162 & 0.569 \\
\hline CR & $7.2(2.7)$ & $7.1(2.5)$ & 0.170 & 0.865 \\
\hline \multicolumn{5}{|c|}{ Executive functions } \\
\hline SCW & $37.4(11.4)$ & $42.8(9.9)$ & -2.217 & 0.030 \\
\hline VF & $16.3(5.4)$ & $17.0(4.9)$ & -0.600 & 0.550 \\
\hline \multicolumn{5}{|c|}{ Visuospatial abilities } \\
\hline $\mathrm{CP}$ & $10.3(1.4)$ & $10.6(0.7)$ & -1.1485 & 0.143 \\
\hline CLOX2 & $14.5(1.1)$ & $14.9(0.3)$ & -1.931 & 0.060 \\
\hline
\end{tabular}

Data are presented as mean (standard deviation) for continuous variables and number (\%) for categorical variables.

${ }^{*} \mathrm{p}<0.05$, independent $\mathrm{t}$-test or chi square test. ${ }^{\dagger} \mathrm{p}<0.0063$, independent $\mathrm{t}$ test with Bonferroni correction.

BMI: body mass index, AHI: apnea-hypopnea index, MMSE-KC: mini-mental state examination in the Korean version of the CERAD assessment packet, DSF: digit span forward, DSB: digit span backward, WLR: word list recall, CR: constructional recall, SCW: Stroop colorword, VF: verbal fluency, CP: constructional praxis, CLOX: executive clock drawing task, OSA: obstructive sleep apnea. 
Table 2. Baseline clinical characteristics and polysomnographic data between non-treated subjects and treated subjects

\begin{tabular}{|c|c|c|c|c|}
\hline & Non-treated OSA $(n=13)$ & Treated OSA $(n=28)$ & $\mathrm{t} / \mathrm{X}^{2}$ & $\mathrm{p}$-value \\
\hline Age (years) & $69.6(5.5)$ & $65.1(4.5)$ & 2.787 & $0.008^{*}$ \\
\hline Gender (male) & $8(61.5 \%)$ & $20(71.4 \%)$ & 2 & 0.818 \\
\hline Education (years) & $11.2(5.5)$ & $14.8(4.0)$ & -2.419 & $0.020^{*}$ \\
\hline \multicolumn{5}{|c|}{ Polysomnographic parameters (pre-treatment) } \\
\hline Total sleep time (min) & $375(54.8)$ & $352(43.8)$ & 1.384 & 0.389 \\
\hline Mean global AHI (events/hr) & $46.8(9.5)$ & $51.7(18.0)$ & -1.127 & 0.267 \\
\hline Mean desaturation index & $39.0(11.6)$ & $42.1(21.7)$ & -0.592 & 0.558 \\
\hline Mean time $\mathrm{SpO}_{2}<90 \%$ & $9.5(9.9)$ & $14.9(19.4)$ & -0.933 & 0.356 \\
\hline Mean $\mathrm{SpO}_{2}$ & $94.0(1.9)$ & $93.2(2.6)$ & 0.992 & 0.327 \\
\hline Mean minimum $\mathrm{SpO}_{2}$ & $74.0(7.8)$ & $77.8(6.8)$ & -1.576 & 0.123 \\
\hline Epworth scale score & $10.0(4.5)$ & $8.9(6.0)$ & 0.548 & 0.588 \\
\hline $\operatorname{BMI}\left(\mathrm{kg} / \mathrm{m}^{2}\right)$ & $24.3(2.8)$ & $25.8(3.6)$ & -1.295 & 0.203 \\
\hline CPAP compliance $(\%)$ & & $77.0(0.14)$ & & \\
\hline $\mathrm{CPAP}$ pressure $\left(\mathrm{cmH}_{2} \mathrm{O}\right)$ & & $9.75(2.37)$ & & \\
\hline Follow up duration (days) & $169.2(40.7)$ & $179.4(111.5)$ & 3.565 & 0.753 \\
\hline
\end{tabular}

Data are presented as mean (standard deviation) for continuous variables and number (\%) for categorical variables.

${ }^{*} \mathrm{p}<0.05$, t-test or chi square test.

AHI: apnea-hypopnea index, $\mathrm{SpO}_{2}$ : saturation percentage of oxygen, BMI: body mass index, CPAP: continuous positive air pressure, OSA: obstructive sleep apnea.

Table 3. Comparison of neuropsychological parameters between treated and non-treated OSA subjects after CPAP treatment

\begin{tabular}{|c|c|c|c|c|c|c|c|}
\hline & \multicolumn{2}{|c|}{ Non-treated OSA $(n=13)$} & \multicolumn{2}{|c|}{ Treated OSA $(n=28)$} & \multicolumn{3}{|c|}{ p-value } \\
\hline & Pre & Post & Pre & Post & Time & Time $\times$ Group & Group \\
\hline MMSE-KC & $27.1(0.84)$ & $27.4(0.73)$ & $27.0(0.54)$ & $27.2(0.47)$ & 0.657 & 0.860 & 0.871 \\
\hline \multicolumn{8}{|l|}{ Memory } \\
\hline DSF & $6.4(0.43)$ & $6.3(0.42)$ & $6.6(0.28)$ & $6.5(0.28)$ & 0.512 & 0.825 & 0.678 \\
\hline DSB & $4.6(0.4)$ & $4.3(0.38)$ & $4(0.26)$ & $4.3(0.25)$ & 0.471 & 0.196 & 0.551 \\
\hline WLR & $6.7(0.58)$ & $7.4(0.57)$ & $5.6(0.38)$ & $6.4(0.37)$ & $0.009^{*}$ & 0.677 & 0.127 \\
\hline CR & $7.3(0.8)$ & $7.9(0.66)$ & $7.2(0.52)$ & $7.7(0.43)$ & 0.073 & 0.960 & 0.850 \\
\hline \multicolumn{8}{|c|}{ Executive functions } \\
\hline SCW & $42.2(3.34)$ & $37.2(2.76)$ & $35.5(2.04)$ & $39.6(1.69)$ & $0.044^{*}$ & $0.023^{*}$ & 0.502 \\
\hline VF & $16.0(1.54)$ & $17.4(1.43)$ & $16.5(1.00)$ & $18.1(0.93)$ & 0.237 & 0.850 & 0.720 \\
\hline \multicolumn{8}{|c|}{ Visuospatial abilities } \\
\hline $\mathrm{CP}$ & $10.3(0.33)$ & $10.4(0.33)$ & $10.3(0.22)$ & $10.3(0.22)$ & 0.351 & 0.754 & 0.993 \\
\hline CLOX & $14.5(0.29)$ & $14.6(0.58)$ & $14.6(0.18)$ & $14.3(0.36)$ & 0.984 & 0.457 & 0.944 \\
\hline
\end{tabular}

Data are presented as the age and education adjusted estimated mean (standard error).

${ }^{*} \mathrm{p}<0.05$, generalized linear model repeated measure ANOVA.

MMSE-KC: mini-mental state examination in the Korean version of the CERAD assessment packet, DSF: digit span forward, DSB: digit span backward, WLR: word list recall, CR: constructional recall, SCW: Stroop color-word, VF: verbal fluency, CP: constructional praxis, CLOX: executive clock drawing task, OSA: obstructive sleep apnea, CPAP: continuous positive airway pressure.

\section{DISCUSSION}

The aim of this study was to assess cognitive function in moderate to severe OSA subjects based on AASM rules, and improvement of cognitive impairment after short term CPAP treatment. In comparison of characteristics, non-treated subjects presented older age and lower levels of education. Polysomnographic parameters demonstrated no difference in both groups. At baseline, mild to severe OSA patients reported higher deficit of attention and working memory than normal 
healthy controls. Despite several inconsistent findings on the effect of OSA on memory functioning, delayed recall memory might be moderately impaired, ${ }^{6}$ and the domain of attention or executive functioning is substantially affected by OSA.$^{24} \mathrm{We}$ previously reported decline of delayed recall and executive function in 42 OSA patients, as compared to 21 normal healthy subjects, indicating that cognition impairments are associated with severe OSA in elderly adults. ${ }^{25}$ However, present samples had higher education years and were younger in age.

The cause of cognitive impairment in OSA patients is under discussion. Recent studies suggest that the decline of executive functions and visuospatial function might be related to severity of hypoxemia; whereas, the attention and memory deficit might be due to excessive daytime sleepiness resulting from sleep fragmentation. ${ }^{26}$ Although correlation analysis in this study was not done among daytime somnolence, measures of nocturnal hypoxemia and results of cognitions, frequent sleep fragmentation might be more effective on attention functioning in OSA groups as comparison of AHI.

After CPAP treatment, alteration of cognition was found in delayed recall memory and executive function overtime in each subjects. However, considering effect of time and groups, only Stroop color word test score was significantly changed in treated subjects, suggestive of improved executive function . Results of several studies are in agreement with improvement of executive dysfunction on CPAP treatment for OSA patients. ${ }^{16,27} \mathrm{Ce}-$ rebral cortex and subcortical structures are responsible for cognitive function. Particularly, frontal lobe and hippocampus of the brain has a major role in cognition. ${ }^{14}$ Various different mechanisms of cognitive impairment in OSA might be involved in sleep fragmentation, brain cell deficits due to hypoxemia, cerebral vascular alteration, sleep deprivation and inflammatory processes. ${ }^{24} \mathrm{CPAP}$ normalized both sleep disruption and oxygen desaturation in OSA patients. Clinical studies and basic research of the executive domain indicate that sleep fragmentation might preferentially affect frontal lobe by interference of the normal restorative sleep, while neuronal cell changes in the prefrontal cortex result from hypoxemia. ${ }^{28}$ Therefore, our findings of improved executive function with CPAP might corroborate this model.

Unlike majority findings of improved attention function with CPAP therapy, ${ }^{24}$ no alteration in attention functioning was found in the present study. The lack of alteration might be due to sample characteristics. In this study, OSA subjects had a significant decline of attention, as compared to normal control at baseline. It is likely that impairment of attention function was uncorrectable by CPAP. In addition, early studies suggested that memory performance might be reversible with optimal hours per days of PAP treatment. ${ }^{29}$ Within treated subjects in our study, CPAP compliance showed $77 \%$ with CPAP in $\geq 4$ hours during total follow up days. As results of multisite study, daytime sleepiness could improve with as few as 4 hours of CPAP use per night. ${ }^{30}$ Longer daily CPAP might be more effective for improvement of cognition. Hippocampal structure for memory is the susceptible region to hypoxia-ischemia perturbation in the elderly. ${ }^{11}$ On the one hand, compliance might be another important factor for duration of CPAP treatment. Effect on cognition after 1-7 days of CPAP use, are conflicting. ${ }^{31}$ But overall, a positive association was reported between using CPAP > 2 week and improvement of cognitive performance. ${ }^{16,18}$ However, a long term efficacy study in 1098 participants, indicated cognition improvement at 2 months after CPAP treatment, not 6 month. ${ }^{13}$ Previous findings showed inconsistency between periods of CPAP use and cognition improvement. Although our subjects had about 3 months of CPAP treatment, greater deviation of use duration was found within treated OSA subjects, which might limit accurate assessment of cognition in response to CPAP. Thus, further study is required to evaluate CPAP treatment with better compliance with longer duration CPAP per night and days of using CPAP.

This study had several limitations. Small number of samples was included. Despite no difference in statistical analysis of clinical characteristics, relatively small number of non-treated subjects participated than treated subjects. To determine the effect of CPAP on cognitive improvement in elderly OSA, it is necessary to differentiate CPAP treated subject according to $\mathrm{CPAP}$ compliance such as good CPAP compliance group and poor CPAP compliance group, which was beyond the scope of our study Additionally, more periodic evaluation of cognitive functions was not conducted. Elderly persons experience difficulty in full battery neurocognitive test and prolonged CPAP treatments. Nevertheless, we evaluated CPAP effect on improvement of cognition with full batteries of cognitive function in the Korean elderly individuals. OSA patients had decline of attention functioning. CPAP treatment showed improvement of executive functioning about 3 months later. Future research for better CPAP compliance including duration of CPAP, applying hours per day and optimal pressure to achieve improved cognition might be needed in the OSA patients.

\section{Conflicts of Interest}

The authors have no financial conflicts of interest.

\section{REFERENCES}

1. Sullivan CE, Issa FG. Pathophysiological mechanisms in obstructive sleep apnea. Sleep 1980;3:235-46.

2. Young T, Palta M, Dempsey J, Skatrud J, Weber S, Badr S. The occurrence of sleep-disordered breathing among middle-aged adults. N Engl J Med 1993;328:1230-5.

3. Dewan NA, Nieto FJ, Somers VK. Intermittent hypoxemia and OSA: implications for comorbidities. Chest 2015;147:266-74.

4. Paiva T, Attarian H. Obstructive sleep apnea and other sleep-related syndromes. Handb Clin Neurol 2014;119:251-71.

5. Sforza E, Roche F, Thomas-Anterion C, Kerleroux J, Beauchet O, Celle $\mathrm{S}$, et al. Cognitive function and sleep related breathing disorders in a healthy elderly population: the SYNAPSE study. Sleep 2010;33:515-21.

6. Beebe DW, Groesz L, Wells C, Nichols A, McGee K. The neuropsycho- 
logical effects of obstructive sleep apnea: a meta-analysis of norm-referenced and case-controlled data. Sleep 2003;26:298-307.

7. Naismith S, Winter V, Gotsopoulos H, Hickie I, Cistulli P. Neurobehavioral functioning in obstructive sleep apnea: differential effects of sleep quality, hypoxemia and subjective sleepiness. J Clin Exp Neuropsychol 2004;26:43-54.

8. Ferini-Strambi L, Marelli S, Galbiati A, Castronovo C. Effects of continuous positive airway pressure on cognitition and neuroimaging data in sleep apnea. Int J Psychophysiol 2013;89:203-12.

9. Alchanatis M, Zias N, Deligiorgis N, Liappas I, Chroneou A, Soldatos C, et al. Comparison of cognitive performance among different age groups in patients with obstructive sleep apnea. Sleep Breath 2008;12:17-24.

10. Silva EJ, Wang W, Ronda JM, Wyatt JK, Duffy JF. Circadian and wakedependent influences on subjective sleepiness, cognitive throughput, and reaction time performance in older and young adults. Sleep 2010; 33:481-90.

11. Gale SD, Hopkins RO. Effects of hypoxia on the brain: neuroimaging and neuropsychological findings following carbon monoxide poisoning and obstructive sleep apnea. J Int Neuropsychol Soc 2004;10:60-71.

12. Aloia MS, Ilniczky N, Di Dio P, Perlis ML, Greenblatt DW, Giles DE. Neuropsychological changes and treatment compliance in older adults with sleep apnea. J Psychosom Res 2003;54:71-6.

13. Kushida CA, Nichols DA, Holmes TH, Quan SF, Walsh JK, Gottlieb DJ, et al. Effects of continuous positive airway pressure on neurocognitive function in obstructive sleep apnea patients: The Apnea Positive Pressure Long-term Efficacy Study (APPLES). Sleep 2012;35:1593-602.

14. Canessa N, Castronovo V, Cappa SF, Aloia MS, Marelli S, Falini A, et al. Obstructive sleep apnea: brain structural changes and neurocognitive function before and after treatment. Am J Respir Crit Care Med 2011; 183:1419-26.

15. Joseph S, Zuriqat M, Husari A. Sustained improvement in cognitive and emotional status of apneic patients after prolonged treatment with positive airway pressure. South Med J 2009;102:589-94.

16. Ferini-Strambi L, Baietto C, Di Gioia MR, Castaldi P, Castronovo C, Zucconi M, et al. Cognitive dysfunction in patients with obstructive sleep apnea (OSA): partial reversibility after continuous positive airway pressure (CPAP). Brain Res Bull 2003;61:87-92.

17. Crawford-Achour E, Dauphinot V, Martin MS, Tardy M, Gonthier R, Barthelemy JC, et al. Protective Effect of Long-Term CPAP Therapy on Cognitive Performance in Elderly Patients with Severe OSA: The PROOF Study. J Clin Sleep Med 2015;11:519-24.

18. Gutiérrez Iglesias B, Jacas Escarceller C, Bardés Robles I, Cambrodi Masip R, Romero Santo-Tomás O, Pujadas Navinés F, et al. Effectiveness of 6 -months continuous positive airway pressure treactment in OSAS-re- lated cognitive deficit in older adults. Behav Neurol 2013;26:191-4.

19. American Academy of Sleep Medicine. International Classification of Sleep Disorders: Diagnostic and Coding Manual. 2nd ed. Westchester, IL: American Academy of Sleep Medicine, 2005;148-52.

20. Rechtschaffen A, Kales AA. A manual of standardized terminology, techniques and scoring system for sleep stages of human subject. Bethesda: National Institute of Neurological Disease and Blindness, 1968.

21. Sleep-related breathing disorders in adults: recommendations for syndrome definition and measurement techniques in clinical research. The Report of an American Academy of Sleep Medicine Task Force. Sleep 1999;22:667-89.

22. Lee JH, Lee KU, Lee DY, Kim KW, Jhoo JH, Kim JH, et al. Development of the Korean version of the Consortium to Establish a Registry for Alzheimer's Disease Assessment Packet (CERAD-K): clinical and neuropsychological assessment batteries. J Gerontol B Psychol Sci Soc Sci 2002;57:P47-53.

23. Seo EH, Lee DY, Choo IH, Kim SG, Kim KW, Youn JC, et al. Normative study of the Stroop Color and Word Test in an educationally diverse elderly population. Int J Geriatr Psychiatry 2008;23:1020-7.

24. Matthews EE, Aloia MS. Cognitive recovery following positive airway pressure (PAP) in sleep apnea. Prog Brain Res 2011;190:71-88.

25. Ju G, Yoon IY, Lee SD, Kim TH, Choe JY, Kim KW. Effects of sleep apnea syndrome on delayed memory and executive function in elderly adults. $J$ Am Geriatr Soc 2012;60:1099-103.

26. Lal C, Strange C, Bachman D. Neurocognitive impairment in obstructive sleep apnea. Chest 2012;141:1601-10.

27. Olaithe M, Bucks RS. Executive dysfunction in OSA before and after treatment: a meta-analysis. Sleep 2013;36:1297-305.

28. Beebe DW, Gozal D. Obstructive sleep apnea and the prefrontal cortex: towards a comprehensive model linking nocturnal upper airway obstruction to daytime cognitive and behavioral deficits. J Sleep Res 2002; 11:1-16.

29. Zimmerman ME, Arnedt JT, Stanchina M, Millman RP, Aloia MS. Normalization of memory performance and positive airway pressure adherence in memory-impaired patients with obstructive sleep apnea. Chest 2006;130:1772-8.

30. Weaver TE, Maislin G, Dinges DF, Bloxham T, George CF, Greenberg $\mathrm{H}$, et al. Relationship between hours of CPAP use and achieving normal levels of sleepiness and daily functioning. Sleep 2007;30:711-9.

31. Bardwell WA, Ancoli-Israel S, Berry CC, Dimsdale JE. Neuropsychological effects of one-week continuous positive airway pressure treatment in patients with obstructive sleep apnea: a placebo-controlled study. Psychosom Med 2001;63:579-84. 